\title{
Construction of a self-cloning system in the unicellular green alga Pseudochoricystis ellipsoidea
}

\author{
Yuki Kasai ${ }^{i^{*}}$, Kohei Oshima', Fukiko Ikeda ${ }^{1}$, Jun Abe ${ }^{1}$, Yuya Yoshimitsu ${ }^{1,2}$ and Shigeaki Harayama ${ }^{1}$
}

\begin{abstract}
Background: Microalgae have received considerable interest as a source of biofuel production. The unicellular green alga Pseudochoricystis ellipsoidea (non-validated scientific name) strain Obi appears to be suitable for large-scale cultivation in outdoor open ponds for biodiesel production because it accumulates lipids to more than $30 \%$ of dry cell weight under nitrogen-depleted conditions. It also grows rapidly under acidic conditions at which most protozoan grazers of microalgae may not be tolerant. The lipid productivity of this alga could be improved using genetic engineering techniques; however, genetically modified organisms are the subject of regulation by specific laws. Therefore, the aim of this study was to develop a self-cloning-based positive selection system for the breeding of $P$. ellipsoidea.
\end{abstract}

Results: In this study, uracil auxotrophic mutants were isolated after the mutagenesis of $P$. ellipsoidea using either ultraviolet light or a transcription activator-like effector nuclease (TALEN) system. The cDNA of the uridine monophosphate synthase gene (PeUMPS) of $P$. ellipsoidea was cloned downstream of the promoter of either a beta-tubulin gene (PeTUBULIN1) or the gene for the small subunit of ribulose 1,5-bisphosphate carboxylase/oxygenase (PeRBCS) to construct the PUT1 or PUT2 plasmid, respectively. These constructs were introduced into uracil auxotroph strains, and genetically complementary transformants were isolated successfully on minimal agar plates. Use of Noble agar as the solidifying agent was essential to avoid the development of false-positive colonies. It took more than 6 weeks for the formation of colonies of pUT1 transformants, whereas pUT2 transformants formed colonies in 2 weeks. Real-time PCR revealed that there were more PeUMPS transcripts in pUT2 transformants than in pUT1 transformants. Uracil synthesis (Ura ${ }^{+}$) transformants were also obtained using a gene cassette consisting solely of PeUMPS flanked by the PeRBCS promoter and terminator.

Conclusions: A self-cloning-based positive selection system for the genetic transformation of $P$. ellipsoidea was developed. Self-cloned P. ellipsoidea strains will require less-stringent containment measures for large-scale outdoor cultivation.

Keywords: Pseudochoricystis ellipsoidea, Green algae, Microalgae, Self-cloning, Uracil auxotroph, Genetic transformation, Molecular breeding

\section{Background}

Microalgae have received considerable interest as a source of biofuel production. In particular, the biodiesel produced by microalgal biomass may be a viable alternative to petroleum-based and land-plant-based fuels because microalgae have several advantages over terrestrial plants for biofuel production, such as low land requirements and high oil yields [1-3]. Although the production of crude oil

\footnotetext{
* Correspondence: ykasai@kc.chuo-u.ac.jp

${ }^{1}$ Department of Biological Sciences, Faculty of Science and Engineering,

Chuo University, Kasuga 1-13-27, Bunkyo-ku, Tokyo 112-8551, Japan

Full list of author information is available at the end of the article
}

from algal biomass has already been achieved in various pilot-scale facilities, the current production costs of algal oil are not competitive with those of fossil oil [4]. The processes used to produce crude algal oil include cultivation and recovery of algal biomass, oil extraction from the algal biomass, and conversion of fatty acid moieties of the oil to liquid biofuels using either methyl esterification or hydrogenation. Currently, efforts are underway to reduce the capital and operating costs of these processes $[5,6]$. However, significant cost reduction could also be achieved via algae breeding, particularly using genetic engineering techniques $[7,8]$. 
Genetically modified algal strains are subject to laws concerning biosafety and also raise ambiguous public concerns; therefore, molecular breeding using techniques based on self-cloning and/or natural occurrence (cloning DNA from a donor into a recipient, between which the natural exchange of DNA is possible) was proposed as a desirable method to construct recombinant organisms for deliberate release into the environment. In Japan, recombinant microorganisms including microalgae, obtained using self-cloning or natural occurrence are exempt from the restrictions imposed by the Cartagena domestic law $[9,10]$.

In plants, concepts named cisgenesis and intragenesis, which are analogous to those of self-cloning and natural occurrence, have been proposed. In cisgenesis and intragenesis, fragments of non-recombinant and recombinant DNA, respectively, from a sexually compatible donor organism is introduced into a recipient. Since both cases involve the exchange of DNA between interbreeding groups, the regulatory measures imposed on cisgenesis and intragenesis could be similar to those for conventional breeding. The regulation of intragenic and cisgenic organisms is currently under discussion in many countries, including in the European Union and the United States, and the regulation of these organisms might be relaxed soon [11]. Amid such trends, the development of self-cloning technology is important for algae breeding using outdoor open pond cultivation, which is currently considered the most viable option for the large-scale cultivation of microalgae [12].

Pseudochoricystis ellipsoidea (non-validated scientific name) strain Obi is a unicellular green alga classified within the family of Trebouxiaceae, which accumulates lipids to an amount of more than $30 \%$ of the dry cell weight under nitrogen-depleted conditions [13]. During nitrogen depletion, the amount of neutral lipids increases, whereas chloroplast-specific glycolipids decrease [14]. A peculiar characteristic of this alga is its ability to grow rapidly under acidic conditions below pH 3.5 without reducing the lipid productivity (Kurano et al., in preparation). Since most protozoan grazers of microalgae might not tolerate that $\mathrm{pH}, P$. ellipsoidea might be suitable for large-scale cultivation in outdoor open ponds for biodiesel production. Thus, we developed a method for the genetic transformation of this alga [15] and are using this method to genetically improve the oil productivity of $P$. ellipsoidea.

We were also interested in developing a self-cloningbased positive selection system for the breeding of P. ellipsoidea for two reasons. First, as discussed above, self-cloning is subject to less-stringent regulation [16, 17], and a consensus regarding the deregulation of the deliberate release of self-cloned microalgae is likely to be built among international experts in the near future. If this occurs, a climate could be developed to foster the cultivation of self-cloned microalgae in outdoor open ponds. Second, genetic engineering includes the controlled overexpression and the targeted inactivation of genes, which both require the use of selectable marker genes to acquire desired clones. Since only one marker is currently available in P. ellipsoidea (G418 resistance), auxotrophic markers will serve as additional selectable markers.

In the current study, we report (i) the isolation of uracil-requiring mutants of $P$. ellipsoidea that are defective in uridine monophosphate synthetase (UMPS), (ii) the synthesis of the full-length cDNA for UMPS, and (iii) the complementation of $P$. ellipsoidea uracil auxotrophs by introducing UMPS cDNA. We encountered several problems that were resolved. This information will be useful for individuals interested in constructing self-cloning systems in other microalgae.

\section{Results and discussion}

\section{Isolation of uracil auxotrophs}

During the ultraviolet light (UV)-mediated mutagenesis of P. ellipsoidea cells, the survival of UV-irradiated cells decreased over UV-C irradiation time and reached $0.1 \%$ after 4 min (see Additional file 1). The mutated cells were screened for the uracil-requiring $\left(\mathrm{Ura}^{-}\right)$phenotype on MA5 minimal agar plates containing both 5-fluoroorotic acid (5-FOA) and uracil. Thirty-six 5-FOA-resistant colonies were isolated after a UV-C dose yielded a survival rate of $\sim 50 \%$, and five of these were uracil auxotrophs. Using genomic DNAs isolated from two of the five uracil auxotrophs named strains M1 and M2, DNA fragments encompassing the coding region (CDR) of the gene for UMPS (PeUMPS) were PCR-amplified, and sequenced. Eukaryotic UMPS is a bifunctional enzyme that consists of orotate phosphoribosyltransferase (OPRTase) and orotidine- $5^{\prime}$-phosphate decarboxylase (ODCase), which catalyze the last two steps of the de novo UMP biosynthetic pathway [18]. The DNA sequencing analysis showed that a 5-base insertion and a 27-base deletion were present in PeUMPS in strains M1 and M2, respectively, both in the OPRTase domain. The spontaneous reversion frequency to $\mathrm{Ura}^{+}$was $7.1 \times 10^{-6}$ for strain M1, and less than $10^{-7}$ for strain M2. Because of the instability of the M1 mutation, strain M1 was not characterized further. Whole-genome sequencing of strain M2 covering $99.4 \%$ of the whole genomic sequence of $P$. ellipsoidea strain Obi (Harayama et al., in preparation) revealed the presence of a one-base substitution in an intron of a gene of unknown function in addition to the PeLMPS mutation described above.

Two uracil auxotrophs named strains M3 and M4 were also isolated from a P. ellipsoidea strain Obi derivative carrying two DNA fragments encoding transcription activator-like effector nuclease (TALEN) right and left 
arms that were designed for the mutagenesis of PeUMPS. The M3 and M4 strains were identified after selecting 5-FOA-resistant colonies grown on MA5 minimal agar plates containing uracil and 5-FOA. The M3 and M4 strains had a 29-base insertion and a 192-base deletion, respectively, in the ODCase domain of PeUMPS. However, these mutations were outside the TALEN-target sequence, suggesting that they arose independently from TALEN activity. The frequency of reversion of the two mutants to $\mathrm{Ura}^{+}$was less than $10^{-7}$.

The M3 and M4 strains that carry the TALEN right and left sequences would not be appropriate hosts for self-cloning. Nevertheless, we used these strains together with strain M2 in following complementation analyses because the mutation loci in the M3 and M4 strains were within the OPRTase domain which was different from the mutation locus within the ODCase domain in strain M2.

\section{Cloning and functional analysis of PeUMPS CDNA}

A $1.5-\mathrm{kb}$ PeUMPS cDNA was cloned into pUC119 to construct pUC_cPeUMPS (Fig. 1). The cloned PeUMPS cDNA sequence was $100 \%$ identical to that predicted from the genomic sequence of $P$. ellipsoidea strain Obi.
The pTV-cPeUMPS plasmid (Fig. 1) was constructed to express PeUMPS cDNA in two uracil auxotrophic mutants of Escherichia coli: JD24489 (an OPRTase mutant) and JW1273 (an ODCase mutant). Both E. coli mutants did not grow on glucose M9 minimal medium, whereas clones that possessed pTV-cPeUMPS grew efficiently, suggesting that the PeUMPS cDNA was expressed functionally in E. coli.

\section{Genetic complementation of uracil auxotrophic $P$. ellipsoidea mutants using PeUMPS CDNA}

Plasmid pUT1 containing PeUMPS cDNA flanked by the promoter of PeTUBULIN1 and the terminator of an actin gene (PeACTIN1) (Fig. 1) was introduced into the uracil auxotrophic strain M4, and prototrophic transformants were selected on MA5 minimal medium solidified using Bacto agar (BD Difco). However, prototrophic transformants could not be selected because of the background growth of strain M4 on MA5 minimal agar plates even when M4 cells grown in MA5 medium supplemented with uracil were washed extensively before plating (data not shown). To reduce the background cell growth, both pG418-T1A [15] (Fig. 1) and pUT1 were co-introduced into strain M4, and the resulting G418-

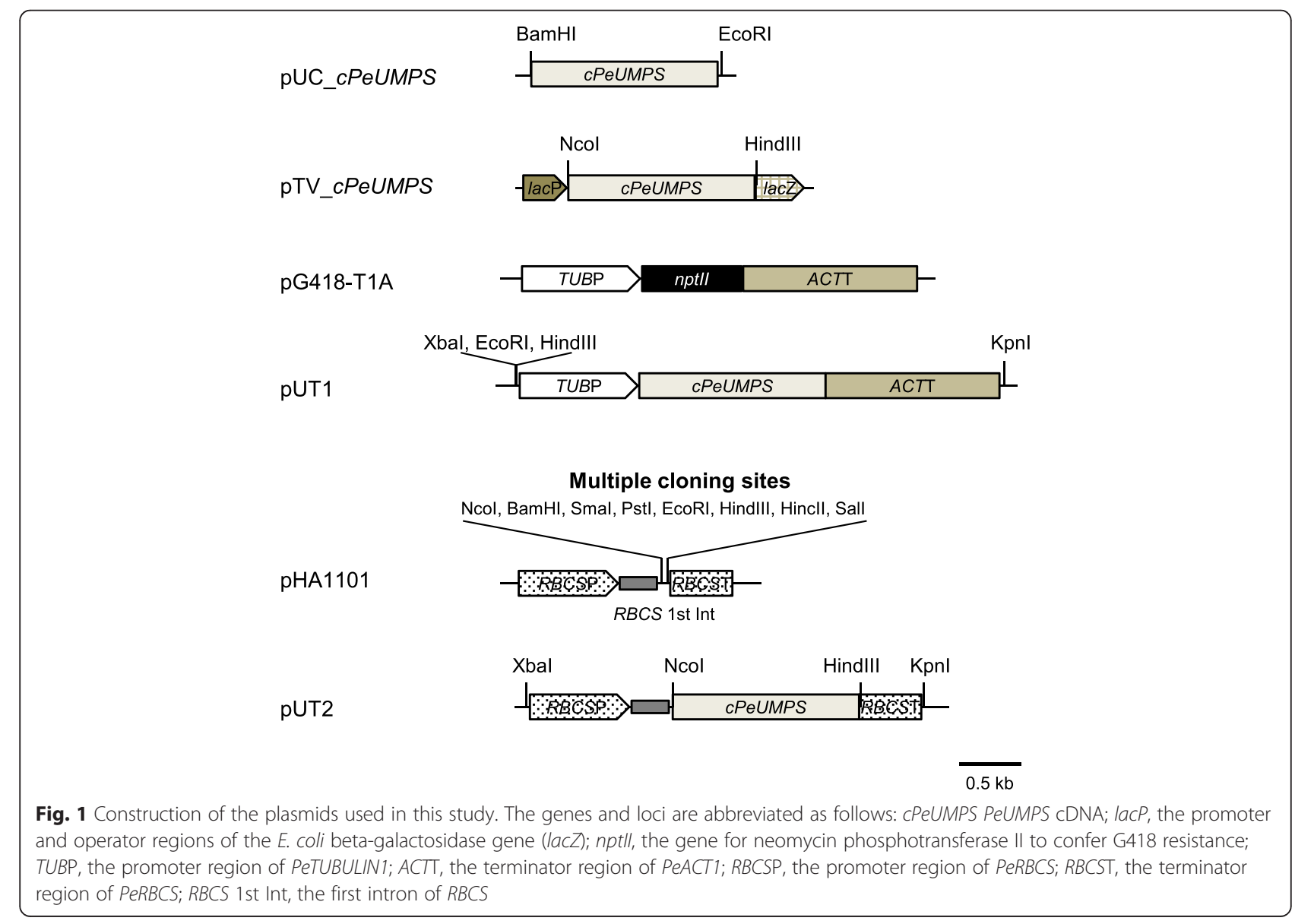


resistant transformants were screened on MA5 minimal agar plates containing G418 and uracil.

Six G418-resistant colonies were obtained, and the existence of pUT1 sequences in the transformants grown on MA5 minimal agar plates containing uracil was examined using PCR with two forward primers (one that hybridized with the 5 -ends of the PeTUBULIN1 promoter, and another that hybridized with the 5 '-ends of the PeUMPS cDNA) and three reverse primers that hybridized the 3 '-end of the PeUMPS cDNA, the middle part of the PeACTIN1 terminator, and the 3 '-end of the PeACTIN1 terminator (Fig. 2). In the genomes of three out of the six transformants (named strains M4-1A, M4-1B, and M4-1C), the DNA region covering the PeTUBULIN1 promoter, PeUMPS cDNA, and the PeACTIN1 terminator was integrated. Conversely, the genome of the transformant named strain M4-1D contained a region covering the PeTUBULIN1 promoter, PeUMPS cDNA, and a 0.4-kb 5'-end of the PeACTIN1 terminator (Fig. 2). The PCR products amplified from the M4-1C genome using the primers Ptub_F and Tact_R were a mixture of four or more DNA fragments (Fig. 2b, lane 2_C), suggesting that the DNA region, the PeTUBULIN1 promoterPeUMPS cDNA-the PeACTIN1 terminator, integrated in the genome of the transgenic M4-1C strain was unstable when grown in the presence of uracil.
When these four PeUMPS-cDNA-positive transgenic strains were streaked onto uracil-free MA5 agar plates, colonies developed only after incubation for $6-8$ weeks at $25{ }^{\circ} \mathrm{C}$. Since strain M4 formed no colony on uracil-free MA5 agar plates after 8 weeks, the introduced PeUMPS cDNA complemented the PeUMPS mutation in strain M4. But the effect was very weak. To shorten the time required for colony formation, MA5 minimal agar plates supplemented with precursors of the UMP biosynthetic pathway (L-glutamate, orotate, and phosphoribosyl pyrophosphate) were prepared, and the growth of the four transgenic strains on the plates was examined. The transgenic strains M4-1C and M4-1D formed colonies in 3 weeks on MA5 minimal agar plates containing orotate; however, the growth improvement by orotate was not significant in the M4-1A and M4-1B strains, which still formed colonies in only 8 weeks. Neither phosphoribosyl pyrophosphate nor L-glutamate improved the growth of any transgenic strains.

To evaluate the correlation between the expression of PeUMPS cDNA and the growth speed, the expression of PeUMPS cDNA in the four transgenic strains was analyzed using real-time PCR and normalized against $18 \mathrm{~S}$ rRNA levels (Fig. 3a). The PCR primers Umps_QRT_F and Umps_QRT_R were used to detect both the expression of transgenic PeUMPS cDNA and the expression of

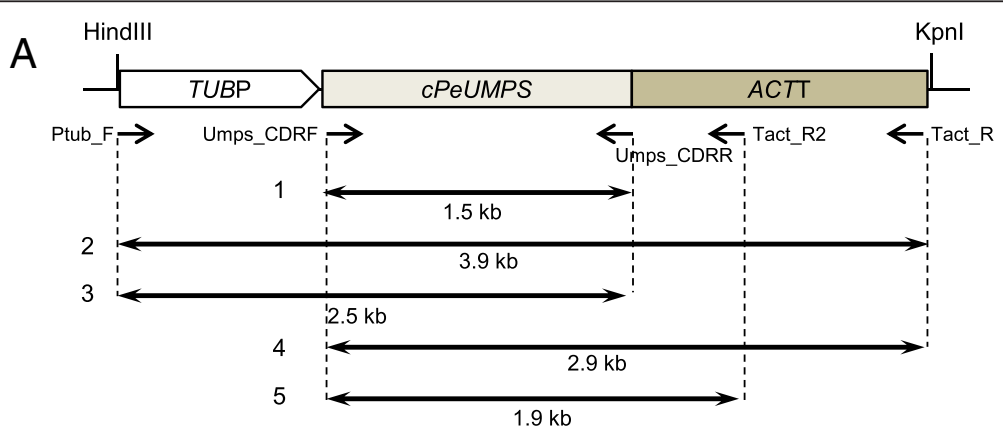

B

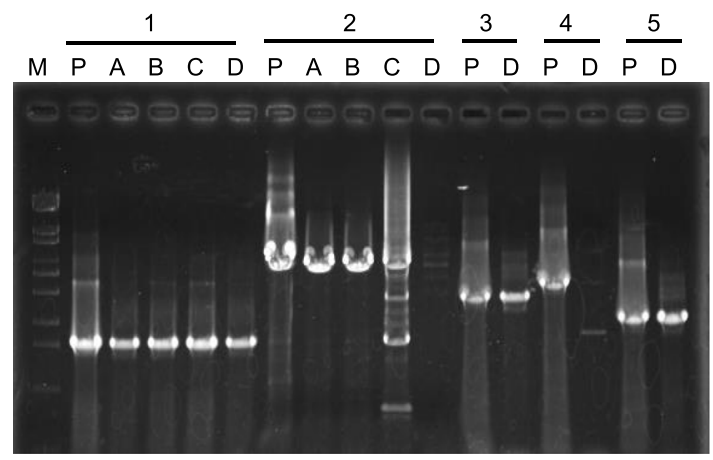

Fig. 2 PCR amplification of the PUT1 sequences integrated into the genomes of transgenic strains. a The pUT1 map: TUBP, the promoter region of PeTUBULIN1; CPEUMPS, the PeUMPS CDNA; ACTT, the terminator region of PeACT1. The positions of the five PCR primers and the PCR products amplified using the primers (1-5) are shown below the pUT1 map. b Agarose gel electrophoresis to verify the presence or absence of PCR products 1-5. M, DNA marker ( $\lambda$-EcoT14 I digest); $P$, pUT1; A, 4 M-1A; $B, 4$ M-1B; $C, 4$ M-1C; $D, 4$ M-1D 

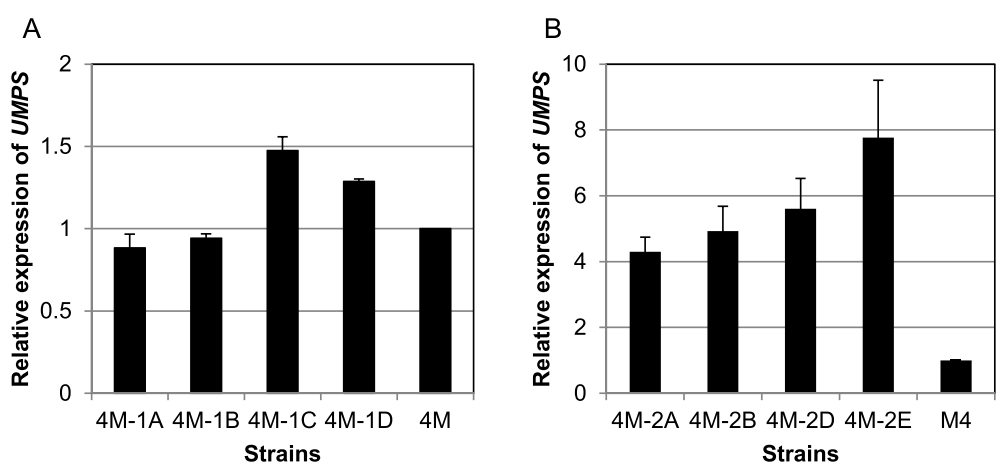

Fig. 3 Real-time PCR analysis of the expression of PeUMPS in (a) pUT1 and (b) pUT2 transgenic strains. 18S rRNA was used as an internal control. Strain M4 is the parental strain of the transgenic strains. Real-time PCR detected expression from the defective PeUMPS in strain M4 in addition to the transgenic PeUMPS cDNA. The data are presented as fold changes in PeUMPS expression normalized to $18 \mathrm{~S}$ rRNA expression in transgenic strains compared with strain M4. Bars represent the standard deviation of three replicates

defective PeUMPS in the chromosome of strain M4. Strain M4-1C, which grew fastest (its colonies were visible in 6 weeks), exhibited a 1.5-fold higher expression than did strain M4. It suggested that the expression of the transgenic PeUMPS cDNA in strain M4-1C was 1.5 -1 -fold of the expression of the original PeUMPS. Similarly, the expression of the transgenic PeUMPS cDNA in strain M4-1D was approximately one quarter of the expression of the original PeUMPS. Strain M4-1D grew faster than strains M4-1A and M4-1B in the presence of orotate. In contrast, no transgene expression was detectable in strains M4-1A and M4-1B, which grew very slowly even in the presence of orotate. These results suggest that the slow growth of the transgenic strains on MA5 minimal agar plates was due to the low expression of the transgenic PeUMPS cDNA.

We next attempted to place the PeUMPS cDNA under the control of a stronger promoter. Whole transcriptome shotgun sequencing (RNA-seq) was applied to $P$. ellipsoidea strain $\mathrm{Obi}$, which revealed that mRNA of the gene for ribulose-1,5-bisphosphate carboxylase/oxygenase small subunit (PeRBCS) was the highest among all nuclear genes and was almost 20 times higher than was that of PeTUBLIN1 (Ide et al., unpublished data). Thus, the PeUMPS cDNA fragment was inserted downstream of the PeRBCS first intron in plasmid pHA1101 to construct plasmid pUT2 (Fig. 1). The pUT2 and pG418-T1A plasmids were then used to co-transform strain M4 using particle bombardment. Among the 106 G418-resistant colonies that developed, the PeUMPS cDNA sequence was identified in eight colonies using PCR. To determine the pUT2 sequences that integrated in the genome of these eight transgenic strains, PCR was performed using three primer sets to detect the $5^{\prime}$-end of the PeRBCS promoter, the middle part of the PeUMPS cDNA, and the 3 '-end of the PeRBCS terminator, respectively (Fig. 4). All three regions were detected in five transgenic strains (M4-2A, M4-2B, M4-2C, M4$2 \mathrm{D}$, and $\mathrm{M} 4-2 \mathrm{E})$, whereas the remaining three transgenic strains contained the PeUMPS cDNA and PeRBCS terminator regions, but not the PeRBCS promoter region (data not shown). The PCR products amplified from the genome of strain M4-2A contained multiple fragments, suggesting that a population of strain M4-2A harbored partial deletions in the integrated expression cassette of the PeRBCS promoter-PeUMPS CDNA-the PeRBCS terminator. Thus, the expression cassette in strain M4-2A was unstable to a certain extent in strain M4-2A. Using genomic DNA isolated from strain M4-2C, fragments 2 and 4 were not amplified efficiently. We interpreted this result as suggesting that strain M4-2C did not carry the full-length expression cassette, but instead carries two or more incomplete expression cassettes: at least one covering the PeRBCS promoter-PeUMPS cDNA region, and one covering the 3 '-end PeUMPS cDNA-the PeRBCS terminator region.

Strains M4-2A, M4-2B, M4-2D, and M4-2E grew on MA5 minimal agar plates and formed colonies within 7 days, similar to the wild-type $P$. ellipsoidea strain Obi. However, strain M4-2C grew on MA5 minimal agar plates and formed colonies after only 6 weeks. Strain M4-2C grew slower than other transgenic strains probably because the strain only carried incomplete forms of the gene expression cassette, as described above.

The expression of PeUMPS cDNA in strains M4-2A, M4-2B, M4-2D, and M4-2E were determined using realtime PCR and normalized to $18 \mathrm{~S}$ rRNA (Fig. 3b). The expression of the transgene was three- to seven-fold higher than was the expression of the chromosomal copy of PeUMPS.

Southern blotting of genomic DNAs extracted from strains M4-1A, M4-1B, M4-1C, M4-1D, M4-2A, M4-2B, M4-2D, and M4-2E was performed, and the results are shown in Additional file 2. Southern blotting analysis of 


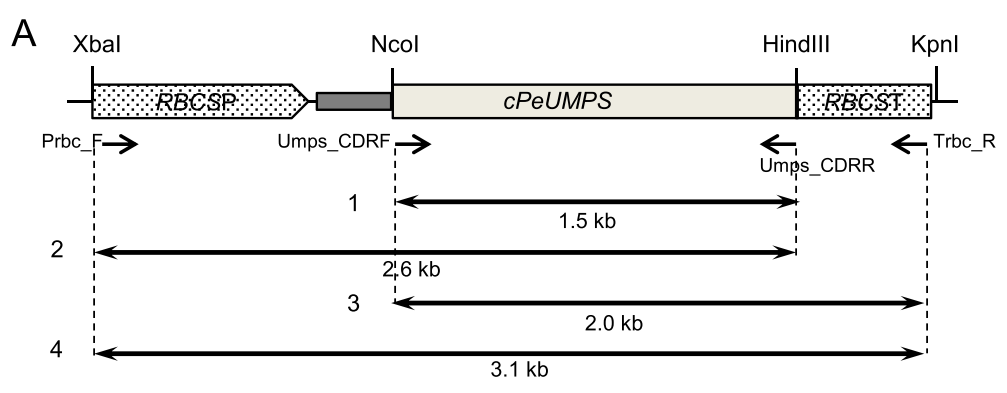

B

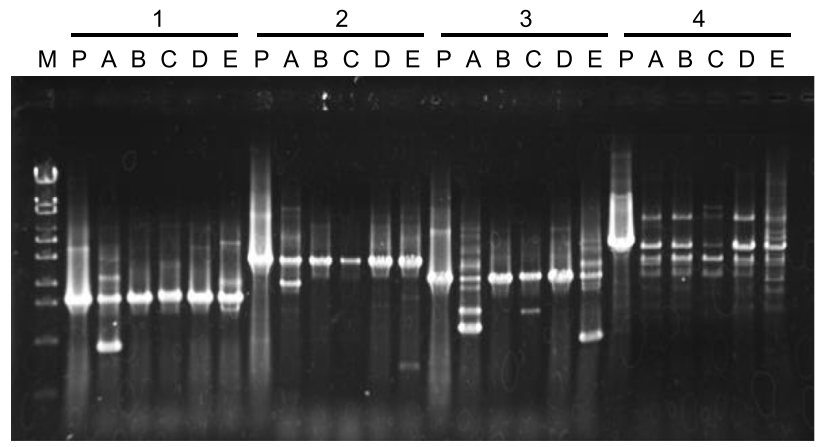

Fig. 4 PCR amplification of the pUT2 sequence that had integrated into the genomes of transgenic strains. a The pUT2 map: RBCSP, the promoter region of PeRBCS; CPeUMPS, PeUMPS CDNA; RBCST, the terminator region of PeRBCS. The positions of the four PCR primers and PCR products amplified using the primers (1-4) are shown below the pUT2 map. b Agarose gel electrophoresis to verify the presence or absence of PCR products 1-4. M, DNA marker ( $\lambda$-EcoT14 I digest); $P$, pUT2; A, 4 M-2A; B, 4 M-2B; C, 4 M-2C; D, 4 M-2D; E, 4 M-2E

genomic DNAs digested with EcoRI should yield two DNA fragments of 8 and $3 \mathrm{~kb}$ (see Additional file 2) originated from the original UMPS gene, that were hybridized to the UMPS cDNA probe. These two bands were detected in all DNA samples, including that from strain M4. In each of the transgenic strains, additional band(s) were detected, and the number of the additional bands indicated the copy number of UMPS cDNAs that integrated into the genome. Thus, the copy numbers were expected to be $2,4,4$, and 4 for strains M4-1A, M4-1B, M4-1C, and M4-1D, respectively, and 7, 1, 4, and 7 for strains M41A, M4-1B, M4-1D, and M4-1E, respectively, based on the Southern blots obtained using EcoRI-digested genomic DNA. Similar results were obtained from Southern blotting performed using XbaI-digested genomic DNA (see Additional file 2). Since strain M4-2B, which carried a single copy of PeUMPS cDNA under the control of the PeRBCS promoter, expressed the transgene at a much higher than did the transgenic strains carrying multiple copies of PeUMPS cDNA under the control of the PeTUBULIN1 promoter, we concluded that the transgene was expressed at significantly higher levels from the PeRBCS promoter compared with the PeTUBULIN1 promoter.

Analysis of the growth of the transgenic strains in MA5 minimal liquid medium showed that transgenic strains containing the pUT1 sequence started to grow with a lag phase of 2-8 days and slower growth rates compared with the wild-type strain ( $P$. ellipsoidea strain Obi) and strain M4 in the presence of $1 \mathrm{mM}$ uracil (Fig. 5a). In contrast, two of the transgenic strains carrying the pUT2 sequence (4 M-2B and $4 \mathrm{M}-2 \mathrm{D})$ grew in MA5 minimal liquid culture at growth rates equivalent to wild-type $P$. ellipsoidea strain $\mathrm{Obi}$, whereas the other two transgenic strains (4 M-2A and $4 \mathrm{M}-2 \mathrm{E}$ ) grew slower than the wild-type strain, despite high levels of transgene expression (Fig. 5b). Although the reason for the slower growth of strains $4 \mathrm{M}-2 \mathrm{~A}$ and $4 \mathrm{M}-2 \mathrm{E}$ is unclear, it is possible that the insertion of a copy of PeUMPS cDNA or the G428-resistance gene into the genomes of these strains disrupted gene(s) that are essential to the rapid growth of $P$. ellipsoidea.

In the studies described above, the PeUMPS cDNA was introduced into strain M4 using a co-transformation technique with pG418-T1A plasmid to first select G418resistant transformants. However, since we demonstrated the practical utility of pUT2 plasmid for complementing the defect in PeUMPS, we next attempted to select $\mathrm{Ura}^{+}$transformants of the uracil auxotroph strains M2, M3, and M4 using this plasmid. As described above, the background growth of these auxotrophs on MA5 minimal agar plates hampered the effective selection of $\mathrm{Ura}^{+}$transformants. Nevertheless, we found that the use of Noble agar (BD Difco) instead of Bacto agar eliminated background growth of the uracil auxotrophic 

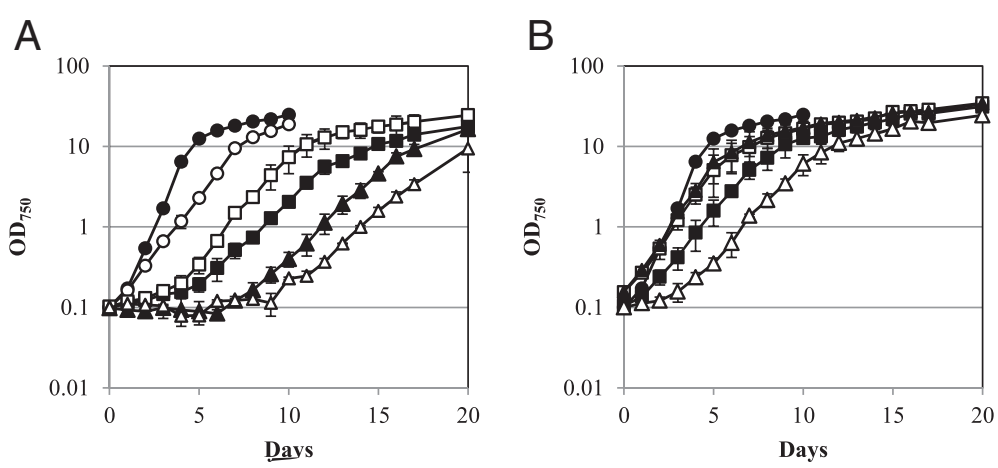

Fig. 5 Growth of transgenic strains in MA5 minimal medium. a P. ellipsoidea strain Obi (closed circles), M4 in the presence of $1 \mathrm{mM}$ uracil (open circles), M4-1A (closed squares), M4-1B (open squares), M4-1C (closed triangles), and M4-1D (open triangles). b P. ellipsoidea strain Obi (closed circles), M4-2A (closed squares), M4-2B (open squares), M4-2D (closed triangles), and M4-2E (open triangles). Experiments were performed in triplicate; bars represent the standard deviation

strains on MA5 minimal agar plates completely. Thus, plasmid pUT2 was introduced in strains M2, M3, and $\mathrm{M} 4$, and $\mathrm{Ura}^{+}$transformants were selected directly on MA5 minimal agar plates solidified using Noble agar (BD Difco). Colonies of $\mathrm{Ura}^{+}$transformants of strains M2, M3, and M4 appeared in 10 days, whereas no colony appeared on the agar plates containing bombarded microcarriers without DNA coating. The transformation rates differed among the uracil auxotrophic strains (Table 1); that of strain M4 was significantly lower than were those of strains M2 and M3.

To establish a method for genetic transformation without introducing foreign nucleic acid sequences (selfcloning), a gene cassette consisting of the PeRBCS promoter-PeUMPS cDNA-the PeRBCS terminator was excised from the pUT2 plasmid using restriction-enzyme digestion, and purified after gel electrophoresis. The linearized gene cassette DNA was then introduced into strain M2 using particle bombardment, and $\mathrm{Ura}^{+}$transformants were selected on MA5. $\mathrm{Ura}^{+}$colonies appeared in 10 days at a frequency of $57.3 \pm 6.9$ transformants $/ \mu g$ DNA. Although this value was half the transformation frequency obtained using pUT2, it was sufficient for the practical use of the gene cassette for the genetic transformation of $\mathrm{Ura}^{-}$strains of $P$. ellipsoidea.

\section{Conclusions}

In this study, a self-cloning-based positive selection system was developed in three distinct steps to obtain transgenic

Table 1 Transformation efficiencies

\begin{tabular}{lll}
\hline Strain & \multicolumn{2}{l}{ Number of transformants/Mg DNA } \\
\hline & pG418-T1A & pUT2 \\
M2 & $42.0 \pm 13.8$ & $122.5 \pm 15.0$ \\
M3 & $19.1 \pm 5.1$ & $123.5 \pm 4.3$ \\
M4 & $18.0 \pm 3.2$ & $15.7 \pm 1.7$ \\
\hline
\end{tabular}

derivatives of $P$. ellipsoidea strain Obi. In the first step, four uracil auxotrophic mutants named strains M1 to M4 were isolated by selecting 5-FOA-resistant derivatives. Strain M2, which was isolated after weak UV mutagenesis, was most convenient as a recipient in genetic transformation because it contained only two mutations: a 27-base deletion in PeUMPS, and a base substitution in an intron of a gene of unknown function. In the second step, UMPS cDNA was cloned and sandwiched between a promoter sequence and a terminator sequence. The PeRBCS promoter was suitable for the transgenic expression of PeUMPS, whereas the PeTUBULIN1 promoter did not support sufficient transgenic expression of PeUMPS. In the third step, the preparation of minimal agar plates using Noble agar was critical for the efficient screening of $\mathrm{Ura}^{+}$transformants. The information obtained in this study will be useful for researchers interested in constructing self-cloning systems in other microalgae.

\section{Methods}

\section{Algal strain and culture conditions}

The algal and bacterial strains used in this study are listed in Table 2. P. ellipsoidea strain Obi (wild-type) and its uracil auxotrophic mutants were cultured in a 120-ml test tube containing $50 \mathrm{ml}$ of MA5 medium [15] or MA5 medium supplemented with $1 \mathrm{mM}$ uracil under continuous illumination with daylight fluorescent tubes $(40 \mathrm{~W}$ FL40S - FR - P, Panasonic) at $100 \mu \mathrm{mol}$ photons $\mathrm{m}^{-2} \mathrm{~s}^{-1}$ in a plant-growth chamber (type \#CLE-303, Tomy). The media were bubbled with $1 \%(v / v) \mathrm{CO}_{2}$ at $25{ }^{\circ} \mathrm{C}$. For agarplate cultivation, the media were solidified using $1.5 \%$ agar (Bacto agar or Noble agar, BD Difco), and the resulting agar plates were incubated in the plant-growth chamber.

\section{Isolation of uracil auxotroph mutants}

Two methods were used to isolate uracil-requiring mutants: UV mutagenesis and TALEN mutagenesis. For UV 
Table 2 List of P. ellipsoidea and E. coli strains used in this study

\begin{tabular}{|c|c|c|}
\hline Strains & Genotype & Source or reference \\
\hline \multicolumn{3}{|c|}{ P. ellipsoidea } \\
\hline Obi & Wild-type & [13] \\
\hline M2 & Carry a 27-base deletion within the OPRTase domain of UMPS & This study \\
\hline M3 & Carry a 29-base insertion within the ODCase domain of UMPS & This study \\
\hline M4 & Carry a 192-base deletion within the ODCase domain of UMPS & This study \\
\hline TAL-1 & $\begin{array}{l}\text { Carry two chromosomally integrated DNA fragments encoding } \\
\text { TALEN left and right arms }\end{array}$ & This study \\
\hline \multicolumn{3}{|l|}{ E. coli } \\
\hline $\mathrm{DH} 5 \mathrm{a}$ & 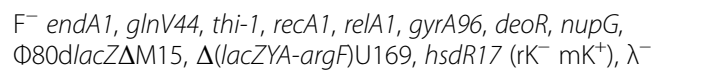 & TOYOBO \\
\hline JD24489 & 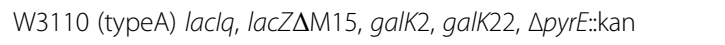 & National Institute of Genetics \\
\hline JW1273 & $\begin{array}{l}\Delta\left(\text { araD-araB) 567, } \Delta l a c Z 4787(:: r r n B-3), \lambda^{-}, \Delta p y r F 789:: k a n, r p h-1,\right. \\
\left.\Delta r h a D-r h a B) 568, \text { hsdR514 (rK } \mathrm{mK}^{-}\right)\end{array}$ & National Institute of Genetics \\
\hline
\end{tabular}

mutagenesis, $P$. ellipsoidea strain Obi cells cultured at an $\mathrm{OD}_{750}$ of 2.2 were collected by centrifugation at $5000 \times g$ for $5 \mathrm{~min}$, and then re-suspended in $50 \mathrm{mM}$ potassium phosphate buffer ( $\mathrm{pH}$ 7.4) to an $\mathrm{OD}_{750}$ of 1 (approximately $2 \times 10^{7}$ cells $\mathrm{ml}^{-1}$ ). Two milliliters of the cell suspension was transferred into an empty Petri dish, stirred using a stirring bar, and irradiated with an ultraviolet (UVC) lamp (15 W GL15, Mitsubishi Electric) for 1-15 min at a distance of $6 \mathrm{~cm}$. Subsequently, the irradiated cells were stored in the dark for $18 \mathrm{~h}$ at $4{ }^{\circ} \mathrm{C}$ to prevent photoreactivation, and then each cell suspension was added to $25 \mathrm{ml}$ of MA5 medium containing $1 \mathrm{mM}$ $\mathrm{Na}_{2} \mathrm{CO}_{3}$ and $1 \mathrm{mM}$ uracil and incubated photosynthetically in the plant-growth chamber until it reached an $\mathrm{OD}_{750}$ of more than 5 to segregate mutated genes. Cells in these cultures were diluted serially, and then spread on MA5 minimal agar plates containing $1 \mathrm{mM}$ uracil and $4.3 \mathrm{mM}$ 5-FOA (Wako Pure Chemical Industries). The agar plates were incubated for 2 weeks in the plantgrowth chamber, and the colonies that grew on the agar plates were picked using sterile toothpicks and streaked on fresh MA5 minimal agar plates containing $1 \mathrm{mM}$ uracil and $3.9 \mathrm{mM}$ 5-FOA for single colony isolation. Each of single colonies that developed on the agar plates was picked using a toothpick and streaked first on an MA5 agar plate and then on an MA5 minimal agar plate containing $1 \mathrm{mM}$ uracil. Among the uracil auxotroph mutants that grew on an MA5 minimal agar plate containing $1 \mathrm{mM}$ uracil but not on an MA5 minimal agar plate, two strains isolated from a culture that had been exposed to a minimal dose of UV irradiation (approximately 1 min of irradiation) were named strains M1 and M2, and maintained.

Strain TAL-1 is a derivative of $P$. ellipsoidea strain Obi that carries in its genome two DNA fragments (TALEN [19-21] right and left arms) that are designed specifically for the mutagenesis of PeUMPS. Two uracil auxotrophs named strains M3 and M4 were isolated from this strain by selecting 5-FOA-resistant colonies on MA5 minimal agar plates containing uracil and 5-FOA.

Genomic DNAs were extracted from these mutants as described by Imamura et al. [15], and the entire PeUMPS sequence was PCR-amplified from each DNA sample using the primers Umps_F (5'-CCACAAAACCC ATTGCCTCAACA-3') and Umps_R (5'-TCCGTGCTG TCTTCCAGGTCTT-3') (Table 3). The PeuMPS sequences in the two mutants were determined using dideoxy chain termination via a commercial service provided by Macrogen Japan Corp.

\section{Cloning of the CDNA encoding UMPS}

Standard molecular procedures were performed as described by Sambrook et al [22]. Total RNA was extracted from $P$. ellipsoidea" strain Obi cells grown to an $\mathrm{OD}_{750}$ of 1.2 using an RNeasy Mini kit (Qiagen), and any remaining genomic DNA was digested using an RNasefree DNase Set (Qiagen) according to the manufacturer's instructions. The RNA concentration was determined from the absorbance at $260 \mathrm{~nm}$, and the RNA integrity was examined using agarose gel electrophoresis. Firststrand cDNA was synthesized using a Transcriptor High Fidelity cDNA Synthesis kit (Roche) with anchored-oligo $(\mathrm{dT})_{18}$ primers according to the manufacturer's instructions. A fragment of PeUMPS cDNA was amplified from a cDNA library using PCR with the primers Umps cDNAF (5'-CGGGATCCCGCTGCATCTTTGCTTTTG TGC-3'; a BamHI site is underlined) and Umps_cDNAR (5'-GGAATTCCCAACCTCATTTGAGAGGCAAT-3'; an EcoRI site is underlined). The resulting amplified fragment was digested using BamHI and EcoRI and ligated into the BamHI and EcoRI sites of pUC119 to create pUCcPeUMPS. The sequence of the resulting PeUMPS 
Table 3 List of PCR primers used in this study

\begin{tabular}{|c|c|c|}
\hline Primer name & Sequence (5' to $\left.3^{\prime}\right)$ & Target \\
\hline Umps_F & CCACAAAACCCATTGCCTCAACA & 5'-end of UMPS \\
\hline Umps_R & TCCGTGCTGTCTTCCAGGTCTT & $3^{\prime}$-end of UMPS \\
\hline Umps_cDNAF & CCAAGCTITGCATGGCATTCAACAAGGTGAA & 5'-end of UMPS CDNA \\
\hline Umps_cDNAR & GGAATTCCCAACCTCATTTGAGAGGCAAT & $3^{\prime}$-end of UMPS CDNA \\
\hline Umps_CDRF & CACCCATGGCAACTTCAACTCCCTCAGT & $5^{\prime}$-end of UMPS CDR \\
\hline Umps_CDRR & CACAAGCTTCATGCCAGCGTGGCCTCATA & 3'-end of UMPS CDR \\
\hline Ptub_F & CCAAGCTTTGCATGGCATTCAACAAGGTGAA & TUBULIN1 promoter \\
\hline Ptub_R_Umps5' & GAGTTGAAGTTGCCATCTTGAACGACTTCCCTCGACAA & $5^{\prime}$-end of UMPS CDR and $3^{\prime}$-end of TUBULIN promoter \\
\hline Umps3'_Tact_F & GCCACGCTGGCATGAGGTGAGCCTGAAACAGCTTTCTG & $3^{\prime}$-end of UMPS CDR and $5^{\prime}$-end of ACT1 terminator \\
\hline Tact_R & TCGGTACCGGTTGTTGAGTTGAGCACTGGAC & $3^{\prime}$-end of $A C T 1$ terminator \\
\hline Tact_R2 & CCCCAGCTGCTACTGCTATC & A middle part of $A C T 1$ terminator \\
\hline Prbc_F & CTCAATCGGCCGATTTCATGCATGA & $5^{\prime}$-end of RBCS promoter \\
\hline Trbc_R & GTGCTGGTCTGTTTCCATGCAGTCAT & $3^{\prime}$-end of $R B C S$ terminator \\
\hline Ptub3'_Umps_CDRF & GGGAAGTCGTTCAAGATGGCAACTTCAACTCCCTCAG & $3^{\prime}$-end of TUBULIN promoter and $5^{\prime}$-end of UMPS CDR \\
\hline Umps_CDRR_Tact5' & TGTITCAGGCTCACCTCATGCCAGCGTGGCCTCATA & $5^{\prime}$-end of ACT1 terminator and $3^{\prime}$-end of UMPS CDR \\
\hline G418F & GATCGGCCATTGAACAAGAT & nptll \\
\hline G418R & GCGATACCGTAAAGCACGAG & nptll \\
\hline Umps_QRT_F & TCTTCGACAAGTGGGATGATGG & Position 908-930 of UMPS CDR \\
\hline Umps_QRT_R & TGTTACCGATGTCCGCAAAC & Position 993-1012 of UMPS CDR \\
\hline 18SrRNA_QRT_F & GGATCAATTGGAGGGCAAGT & Position $627-646$ of $18 \mathrm{~S}$ rRNA \\
\hline 18SrRNA_QRT_R & GCCCGAAATCCAACTACGAG & Position $713-732$ of 185 rRNA \\
\hline
\end{tabular}

cDNA was verified using double-stranded sequencing as described above. To construct a PeUMPS cDNA expression vector in $E$. coli, PCR was performed using the primers Umps_CDRF (5'-CACCCATGGCAACTTCA ACTCCCTCAGT-3'; an Ncol site is underlined) and Umps_CDRR (5'-CACAAGCTTCATGCCAGCGTGG CCTCATA-3'; a HindIII site is underlined). The amplified fragments were then digested using NcoI and HindIII and cloned into the NcoI and HindIII sites of pTV118 (TaKaRa) to create pTV_cPeUMPS. To confirm the functionality of the cloned PeUMPS cDNA, pTV_cPeUMPS was introduced into the uracil auxotrophic E. coli strains JD24489 [W3110 (type A), lacI $^{q}$, lacZ $\Delta \mathrm{M} 15$, galK2, galK22, $\Delta$ pyrE::kan] and JW1273 $\left[\Delta(\right.$ araD-araB $) 567, \Delta$ lacZ4787(::rrnB-3), $\lambda^{-}, \Delta$ pyrF789:: kan, rph-1, $\Delta$ (rhaD-rhaB)568, hsdR514] [23], which were purchased from the National Institute of Genetics of Japan (Mishima). The growth of the E. coli transformants was examined on glucose M9 minimal medium [22].

\section{Construction of the PeUMPS cDNA vectors for expression in $P$. ellipsoidea}

The pUT1 plasmid (NCBI/DDBJ accession number LC016841) carrying the PeUMPS cDNA flanked by the PeTUBULIN1 promoter and the PeACTIN1 terminator was constructed in two steps (see Additional file 3) using
PrimeSTAR MX DNA polymerase (Takara) as described previously [24]. In the first step, three DNA fragments were amplified separately using PCR. Specifically, a 0.9-kb promoter region of PeTUBULIN1 of P. ellipsoidea strain Obi was amplified using primers Ptub_F (5' -CCAAGCTT TGCATGGCATTCAACAAGGTGAA-3'; a HindIII site is underlined) and Ptub_R_Umps5' (5' -GAGTTGAAGT TGCCATCTTGAACGACTTCCCTCGACAA-3'; an adapter sequence for the second-step PCR is underlined), and a 1.5-kb terminator region of PeACTIN1 of P. ellipsoidea strain Obi was amplified using the primers Umps3'_ Tact_F (5'-GCCACGCTGGCATGAGGTGAGCCTGAA ACAGCTTTCTG-3'; an adapter sequence for the secondstep PCR is underlined) and Tact_R (5' ${ }^{\prime}$-TCGGTACC GGTTGTTGAGTTGAGCACTGGAC-3'; a KpnI site is underlined) with pG418-T1A DNA [15] as the template. In parallel, a $1.5-\mathrm{kb}$ PeUMPS cDNA was amplified using the primers Ptub3'_Umps_CDRF (5'-GGGAAGTCGTTCAAG ATGGCAACTTCAACTCCCTCAG-3'; an adapter sequence for the second-step PCR is underlined) and Umps_CDRR_Tact5' (5'-TGTTTCAGGCTCACCTCAT GCCAGCGTGGCCTCATA-3', an adapter sequence for the second-step PCR is underlined) and pUC-cPeUMPS plasmid as the template. In the second step, the three fragments amplified in the first step were assembled into a single fragment using PCR with the three fragment DNAs 
as the templates and the primers Ptub_F and Tact_R. The resulting DNA fragment was purified using a PCR purification kit (Qiagen), digested using HindIII and KpnI, and cloned into the HindIII and KpnI sites of pBluescript II sk (+).

Next, the PeUMPS cDNA was placed between two DNA fragments: one containing the promoter $-5^{\prime}$ untranslated region-first intron region of PeRBCS and the other containing the $3^{\prime}$ untranslated region-terminator sequence of PeRBCS. Briefly, the 1.5-kb PeUMPS cDNA was amplified using PCR with the primers Umps_CDRF and Umps_CDRR with pUC-cPeUMPS DNA as the template. The PCR product was digested using NcoI and HindIII, and then cloned into the NcoI and HindIII sites of pHA1011 (NCBI/DDBJ accession number LC016840) between the promoter and terminator regions of PeRBCS to construct pUT2 (NCBI/DDBJ accession number LC016842).

\section{Transformation of $P$. ellipsoidea cells}

Nuclear transformation was performed using particle bombardment as described previously [15], with slight modifications. Uracil auxotrophic strains were grown to an $\mathrm{OD}_{750}$ of 0.5 in MA5 medium supplemented with 1 $\mathrm{mM}$ uracil, and $1 \mathrm{ml}$ of the culture was spread on an MA5 minimal agar plate containing $1 \mathrm{mM}$ uracil. After drying the surface of the plate, samples were incubated at $25{ }^{\circ} \mathrm{C}$ for 3 days under 12-h light/dark cycles at a light intensity of $50 \mu \mathrm{mol} \mathrm{m} \mathrm{m}^{-2} \mathrm{~s}^{-1}$. Then, the plates were placed under the stopping screen of a Biolistic PDS$1000 / \mathrm{He}$ particle delivery system (Bio-Rad) at a distance of $6 \mathrm{~cm}$. DNA-coated gold particles of either $0.6 \mu \mathrm{m}$ (Bio-Rad) or $0.3 \mu \mathrm{m}$ (Recenttec) diameter were prepared according to the instructions given by Bio-Rad (technical note \# 2453), and the cells on MA5 minimal agar plates were bombarded twice at helium pressures of 1100 or $1550 \mathrm{psi}$, respectively, in vacuo with $125 \mathrm{~mm} \mathrm{Hg}$. After bombardment, the cells were incubated at $25{ }^{\circ} \mathrm{C}$ in the dark for $24 \mathrm{~h}$, and then transferred onto appropriate selection plates.

For the isolation of G418-resistant transformants, cells were suspended in $1 \mathrm{ml}$ of MA5 minimal medium, spread onto MA5 minimal agar plates containing $100 \mu \mathrm{g}$ $\mathrm{ml}^{-1} \mathrm{G} 418$ and $1 \mathrm{mM}$ uracil, and incubated in a $\mathrm{CO}_{2}$ incubator at $5 \%(v / v) \mathrm{CO}_{2}$ and $25{ }^{\circ} \mathrm{C}$ under fluorescence tubes at $50 \mu \mathrm{mol}$ photon $\mathrm{m}^{-2} \mathrm{~s}^{-1}$. The numbers of G418-resistant colonies that developed on the selection plates were counted after incubation for 14 days to calculate the transformation frequencies. Each of the single colonies that developed on the agar plates was streaked onto fresh MA5 minimal agar plates containing $100 \mu \mathrm{g}$ $\mathrm{ml}^{-1} \mathrm{G} 418$ and $1 \mathrm{mM}$ uracil. PCR templates for genotyping the G418-resistant transformants were prepared from cells grown on the agar plates as follows: the cells were suspended in $50 \mu \mathrm{l}$ of TE buffer ( $\mathrm{pH} 8.0$ ), disrupted by freezing in liquid nitrogen and thawing at $70{ }^{\circ} \mathrm{C}$, and cell debris was removed by centrifugation. PCR was then performed to identify G418-resistant clones using the supernatant as the template and the primers $\mathrm{G} 418 \mathrm{~F}$ (5'-GATCGGCCATTGAACAAGAT-3') and G418R (5'-GC GATACCGTAAAGCACGAG-3'). In addition, PCR to detect PeUMPS cDNA was performed using the primers Umps_CDRF and Umps_CDRR. PCR to determine a pUT1 region integrated in the genomes of transgenic strains was performed using either Umps_CDRF or Ptub_F as the forward primer, and the reverse primers Umps CDRR, Tact_R2 (5'-CCCCAGCTGCTACTGCTATC-3'), or Tact_R as shown in Fig. 2. PCR to determine a pUT2 region integrated in the genomes of transgenic strains was performed using Prbc_F (5'-CTCAATCGGCCGATTTCATGCATGA-3') or Umps_CDRF as the forward primer, and Umps_CDRR or Trbc_R (5' -GTGCTGGTCTGTTTC CATGCAGTCAT-3') as the reverse primer, as shown in Fig. 4.

For the direct isolation of $\mathrm{Ura}^{+}$transformants, pUT2 plasmid was introduced into strain M2 using particle bombardment as described above. After bombardment, the cells were spread onto MA5 minimal plates solidified with Noble agar. Each single colony that developed on the Noble agar plates was streaked onto fresh MA5 minimal agar plates, and cells that grew on the plates were used for genotype analyses. The PCR to detect UMPS cDNA in $\mathrm{Ura}^{+}$transformants was performed using the primers Umps_CDRF and Umps_CDRR.

\section{Southern blotting}

Three micrograms of genomic DNA was digested using restriction enzyme EcoRI or XbaI, separated on $0.8 \%$ $(w / v)$ agarose gels, and blotted onto a Hybond-N+ (GE Healthcare, UK) using a standard capillary transfer method with $20 \times$ SSC as the transfer buffer. The blotted filter was baked at $80{ }^{\circ} \mathrm{C}$ for $2 \mathrm{~h}$, and the PeUMPS cDNA fragment was labeled using a DIG High Prime DNA labeling and detection kit (Roche Applied Sciences). Hybridization and signal detection were performed according to the manufacturer's instructions.

\section{Real-time PCR}

Total RNA was extracted from cells grown in MA5 minimal medium to an $\mathrm{OD}_{750}$ of 2.0 using a TRIzol ${ }^{\circ}$ plus RNA purification kit (Ambion), and remaining DNA was digested using a TURBO DNA-free kit (Ambion) according to the manufacturer's instructions. First-strand cDNA was synthesized using a PrimeScript ${ }^{\mathrm{Tw}} \mathrm{RT}$ reagent kit with gDNA Eraser (Perfect Real Time, TaKaRa) and an RT primer mix containing oligo $(\mathrm{dT})_{18}$ and random hexamers. Real-time PCR $\left(95^{\circ} \mathrm{C}\right.$ for $30 \mathrm{~s}$, followed by 40 cycles of $95{ }^{\circ} \mathrm{C}$ for $5 \mathrm{~s}$, and $60{ }^{\circ} \mathrm{C}$ for $30 \mathrm{~s}$ ) to quantify 
PeUMPS cDNA was performed using the primers Umps_QRT_F (5'-TCTTCGACAAGTGGGATGATGG3'; position 908-930 of the 1597-bp UMPS cDNA) and Umps_QRT_R (5'-TGTTACCGATGTCCGCAAAC-3'; position 993-1012 of the 1597-bp UMPS cDNA) on a TaKaRa Thermal Cycler Dice ${ }^{\bullet}$ real-time system II using SYBR $^{\circ}$ Premix Ex Taq ${ }^{\text {Ta }}$ II (Tli RNaseH Plus, TaKaRa) according to the manufacturer's instructions. The levels of $18 \mathrm{~S}$ rRNA were also determined as an internal control using the primers 18SrRNA_QRT_F (5'-GGATCAAT TGGAGGGCAAGT-3'; position 627-646 of the 1899-bp $18 \mathrm{~S}$ rRNA) and 18SrRNA_QRT_R (5'-GCCCGAAATC CAACTACGAG-3'; position 713-732 of the 1899-bp 18S rRNA). The melting curves for each PCR product were determined by measuring the decrease in fluorescence with increasing temperature from 60 to $95{ }^{\circ} \mathrm{C}$. Real-time PCR without a template was also performed in each experiment as a negative control.

\section{Accession numbers}

Sequence data from this study can be found in the DDBJ/NCBI data libraries under the accession numbers LC016838 (PeUMPS), LC016839 (PeUMPS_cDNA), LC016841 (pUT1), LC016840 (pHA1011), and LC016842 (pUT2).

\section{Additional files}

Additional file 1: UV radiation survival curves. UV radiation survival curves for $P$. ellipsoidea strain Obi.

Additional file 2: Southern blotting. (A) The restriction sites in the 13-kb genomic DNA region comprising PeUMPS are shown. (B) The restriction sites in PUT1 and PUT2 are shown. (C) Southern blotting of genomic DNAs isolated from eight transgenic strains. Genomic DNA was digested using EcoRI or Xbal, and hybridized with a digoxigenin-labeled PeUMPS CDNA. Lane PC, pUT2 plasmid digested with Hindlll and Xbal as a positive control; lane 1, M4; lanes 2-5, representative transgenic M4 with pUT1 (M4-1D, M4-1C, M4-1A, and M4-1B); lanes 6-9, representative transgenic M4 with pUT2 (M4-2A, M4-2B, M4-2D, and M4-2E). The numbers on the left and right sides of the gels indicate molecular size expressed in kilobase pairs. Arrowheads indicate restriction fragments containing the chromosomal UMPS sequence.

Additional file 3: Construction of pUT1 in two-step PCR. Ptub_F and Tact_R carry HindIII and Kpnl site, respectively, that were used for the cloning into pBluescript II sk(+).

\section{Abbreviations}

5-FOA: 5-fluoroorotic acid; CDR: coding region; ODCase: orotidine-5'-phosphate decarboxylase; OPRTase: oroate phosphoribosyltransferase; PeACTIN1: actin gene; PeRBCS: 1,5-bisphosphate carboxylase/oxygenase small subunit gene; PeTUBULIN1: beta-tubulin gene; PeUMPS: uridine monophosphate synthase gene; TALEN: transcription activator-like effector nuclease; UMPS: uridine monophosphate synthetase; Ura+: uracil synthesis; UV: ultraviolet light.

\section{Competing interests}

The authors declare that they have no competing interests.

\section{Authors' contributions}

YK designed the experiments, carried out molecular genetic studies, drafted this manuscript and revised it critically for important intellectual content. $\mathrm{KO}$ carried out UV mutagenesis and molecular genetic studies and helped to draft the manuscript. Fl designed and carried out genetic transformation, and helped to draft the manuscript. JA designed and carried out molecular genetic studies including genetic transformation and helped to draft the manuscript. YY designed and carried out TALEN mutagenesis and molecular genetic studies, analyzed the data, and helped to draft the manuscript. SH conceived the study, participated in its design and coordination, and helped to draft the manuscript. All authors read and approved the final version of the manuscript.

\section{Acknowledgements}

We thank Ms. Izumi Fukuhara for her advice, Ms. Satsuki Takagi for technical assistance and Dr. Yoko Ide for providing us the genomic sequence of $P$. ellipsoidea strain Obi. This work was supported by New Energy and Industrial Technology Development Organization (NEDO, P11502725-0).

\section{Author details}

'Department of Biological Sciences, Faculty of Science and Engineering, Chuo University, Kasuga 1-13-27, Bunkyo-ku, Tokyo 112-8551, Japan. ${ }^{2}$ Research Laboratories, Denso Corporation, Nisshin, Aichi 470-0111, Japan.

Received: 9 March 2015 Accepted: 19 June 2015

Published online: 30 June 2015

\section{References}

1. Carlsson AS, van Beilen JB, Möller R, Clayton D. Micro- and macro-algae: utility for industrial applications, outputs from the EPOBIO project. In: Bowles D, editor. Newbury (UK): University of York, CPL Press September; 2007. p. 86.

2. Priyadarshani I, Rath B. Commercial and industrial applications of micro algae-a review. J Algal Biomass UtIn. 2012;3:89-100.

3. Yaakob Z, Ali E, Zainal A, Mohamad M, Takriff MS. An overview: biomolecules from microalgae for animal feed and aquaculture. J Biol Res. 2014;21:6.

4. Chisti Y. Constraints to commercialization of algal fuels. J Biotechnol. 2013;167:201-14.

5. Chiaramonti D, Prussi M, Casini D, Tredici MR, Rodolfi L, Bassi N, et al. Review of energy balance in raceway ponds for microalgae cultivation: re-thinking a traditional system is possible. Appl Energy. 2013;102:101-11.

6. Coons JE, Kalb DM, Dale T, Marrone BL. Getting to low-cost algal biofuels: a monograph on conventional and cutting-edge harvesting and extraction technologies. Algal Res. 2014;6:250-70.

7. Gimpel JA, Specht EA, Georgianna DR, Mayfield SP. Advances in microalgae engineering and synthetic biology applications for biofuel production. Curr Opin Chem Biol. 2013;17:489-95.

8. Blatti JL, Michaud J, Burkart MD. Engineering fatty acid biosynthesis in microalgae for sustainable biodiesel. Curr Opin Chem Biol. 2013;17:496-505.

9. Lusser M, Rodríguez-Cerezo E. Comparative regulatory approaches for new plant breeding techniques. -Workshop Proceedings. European Commission. JRC Technical Report EUR 252737 EN. 2012.

10. United States Department of Agriculture. Japan takes step forward to improve its GE product review process. USDA Japan reports. 2014. (usdajapan.org/en/reports/reports2014.html).

11. Holme IB, Wendt T, Holm PB. Intragenesis and cisgenesis as alternatives to transgenic crop development. Plant Biotechnol J. 2013;11:395-407.

12. Brennan $L$, Owende P. Biofuels from microalgae - a review of technologies for production, processing, and extractions of biofuels and co-products. Renew Sust Energ Rev. 2010;14:557-77.

13. Satoh A, Kato M, Yamato K, Ishibashi M, Sekiguchi H, Kurano N, et al. Characterization of the lipid accumulation in a new microalgal species, Pseudochoricystis ellipsoidea (Trebouxiophyceae). J Jpn Inst Energ. 2010;89:909-13.

14. Ito T, Tanaka M, Shinkawa H, Nakada T, Ano Y, Kurano N, et al. Metabolic and morphological changes of an oil accumulating trebouxiophycean alga in nitrogen-deficient conditions. Metabolomics. 2013;9:178-87.

15. Imamura S, Hagiwara D, Suzuki F, Kurano N, Harayama S. Genetic transformation of Pseudochoricystis ellipsoidea, an aliphatic hydrocarbonproducing green alga. J Gen Appl Microbiol. 2012;58:1-10.

16. Hino A. Safety assessment and public concerns for genetically modified food products: the Japanese experience. Toxicol Pathol. 2002;30:126-8.

17. Fischer $S$, Procopio S, Becker T. Self-cloning brewing yeast: a new dimension in beverage production. Eur Food Res Technol. 2013;237:851-63.

18. Traut TW, Jones ME. Uracil metabolism - UMP synthesis from orotic acid or uridine and conversion of uracil to beta-alanine: enzymes and cDNAs. Prog Nucleic Acid Res Mol Biol. 1996;53:1-78. 
19. Christian M, Cermak T, Doyle EL, Schmidt C, Zhang F, Hummel A, et al. Targeting DNA double-strand breaks with TAL effector nucleases. Genetics. 2010;186:757-61.

20. Miller JC, Tan S, Qiao G, Barlow KA, Wang J, Xia DF, et al. A TALE nuclease architecture for efficient genome editing. Nat Biotechnol. 2011;29:143.

21. Wood AJ, Lo T-W, Zeitler B, Pickle CS, Ralston EJ, Lee AH, et al. Targeted genome editing across species using ZFNs and TALENs. Science. 2011;333:307.

22. Sambrook J, Fritsch EF, Maniatis T. Molecular cloning: a laboratory manual. 2nd ed. New York: Cold Spring Harbor Laboratory Press, Cold Spring Harbor; 1989.

23. Baba T, Ara T, Hasegawa M, Takai Y, Okumura Y, Baba M, et al. Construction of Escherichia coli K-12 in-frame, single-gene knockout mutants: the Keio collection. Mol Syst Biol. 2006:2:1-11.

24. Imamura S, Kanesaki Y, Ohnuma M, Inouye T, Sekine $Y$, Fujiwara T, et al. R2R3-type MYB transcription factor, CmMYB1, is a central nitrogen assimilation regulator in Cyanidisoschyzon merolae. Proc Natl Acad Sci U S A. 2009:106:12548-53.

\section{Submit your next manuscript to BioMed Central and take full advantage of:}

- Convenient online submission

- Thorough peer review

- No space constraints or color figure charges

- Immediate publication on acceptance

- Inclusion in PubMed, CAS, Scopus and Google Scholar

- Research which is freely available for redistribution 